\title{
Peristaltic Transport of Magnetohydrodynamic Carreau Nanofluid with Heat and Mass Transfer inside Asymmetric Channel
}

\author{
Nabil T. M. Eldabe1, Osama M. Abo-Seida², Adel A. S. Abo-Seliem³, A. A. ElShekhipy ${ }^{4,5}$, \\ Nada Hegazy ${ }^{*}$ \\ ${ }^{1}$ Mathematics Department, Faculty of Education, Ain Shams University, Cairo, Egypt \\ ${ }^{2}$ Faculty of Computers and Information, Kafrelsheikh University, Kafrelsheikh, Egypt \\ ${ }^{3}$ Mathematics Department, Faculty of Science, Kafrelsheikh University, Kafrelsheikh, Egypt \\ ${ }^{4}$ Mathematics Department, Faculty of Science, Minia University, El-Minia, Egypt \\ ${ }^{5}$ Mathematics Department, Faculty of science, Imam Abdulrahman Bin Faisal University, Al-Dammam, KSA \\ Email:*nadahegazy142@yahoo.com
}

How to cite this paper: Eldabe, N.T.M., Abo-Seida, O.M., Abo-Seliem, A.A.S., ElShakhipy, A.A. and Hegazy, N. (2017) Peristaltic Transport of Magnetohydrodynamic Carreau Nanofluid with Heat and Mass Transfer inside Asymmetric Channel. American Journal of Computational Mathematics, 7, 1-20.

https://doi.org/10.4236/acim.2017.71001

Received: January 27, 2017

Accepted: March 20, 2017

Published: March 23, 2017

Copyright (๑) 2017 by authors and Scientific Research Publishing Inc. This work is licensed under the Creative Commons Attribution International License (CC BY 4.0).

http://creativecommons.org/licenses/by/4.0/ (c) (i) Open Access

\begin{abstract}
In this work, the peristaltic motion of a nano non-Newtonian fluid which obeys Carreau model through a porous medium inside an asymmetric channel is investigated. The hall current effects with Joule heating and viscous dissipation are considered. The problem is modulated mathematically by a set of nonlinear partial differential equations which describe the conservation of mass, momentum, energy and concentration of nanoparticles. The non-dimensional form of these equations is simplified under the assumption of long wavelength and low Reynolds number, and then resulting equations of coupled nonlinear differential equations are tackled numerically with appropriate boundary conditions. Graphical results are presented for dimensionless velocity, temperature, concentration and pressure gradient in order to illustrate the variations of various parameters of this problem on these obtained solutions.
\end{abstract}

\section{Keywords}

Peristaltic Motion, MHD, Carreau Nanofluid, Asymmetric Channel, Porous Medium, Heat and Mass Transfer

\section{Introduction}

Nowadays, the study of nanofluids flow has the interest of researches because of its applications in medicine, biochemistry and industrial engineering. Nanofluids are moderately new category of fluids which consist of a base-fluid with nano-sized particles $(1-100 \mathrm{~nm})$ suspended within them. Choi [1] may be the first author to use the term "nanofluid", where it was reported that one of the prom- 
ising nanofluids applications in heat could transfer enhancement. In [2], Choi et al. showed that the addition of a small amount (less than $1 \%$ by volume) of nanoparticles to conventional heat transfer liquids increase the thermal conductivity of the fluid up to approximately two times. An analysis of nanofluids examined by Buongiorno [3] was induced that this massive increase in the thermal conductivity occurs due to the presence of the Brownian diffusion and the thermophoretic diffusion of the nanoparticles. Recent articles on the nanofluid are cited in ReFS [4] [5] [6] [7] [8].

Peristaltic motion in a channel or tube is considered as a type of flow that has great value in several physiological processes and industries. Peristalsis is a mechanism for mixing and transporting fluids through expansion and contraction of the wave propagation along the channel walls. This mechanism is seen in many biological system such as urine transport from kidney to bladder through the ureter, transport of lymph in the lymphatic vessels, swallowing food through the esophagus, the movement of chime in the gastrointestinal track, ovum movement in the fallopian tube, transport of spermatozoa vasomotion of small blood vessels such as venules and capillaries and blood flow in arteries, transport of corrosive fluids, sanitary fluid transport and blood pumps in heart lung machine etc. This analysis was first investigated by Latham [9]. He presented fluid motion in peristaltic pump and also discussed the characteristic of pressure rise verus flow rate. After the work of Latham [9], Jaffrin and Shapiro [10] investigated the peristaltic pumping. They made the study under the assumption of long wavelength and low Reynolds number approximation. This work was further extended for Newtonian and non-Newtonian fluids with different flow geometries and boundary conditions as elaborated in the references [11]-[17].

Recently, Eldabe et al. [18] have discussed peristaltic transport of a magneto non-Newtonian fluid through a porous medium in a horizontal finite channel. Safia Akram [19] has investigated the effects of nanofluid on peristaltic flow of a Carreau fluid model in an inclined magnetic field. Arshad et al. [20] have analyzed the peristaltic transport of a Carreau fluid in a compliant rectangular duct. Khalid Nowar [21] has studied peristaltic flow of a nanofluid under the effect of Hall current and porous medium. Noreen et al. [22] have examined numerical simulation of peristaltic flow of a Carreau naofluid in an asymmetric channel. Tasawar et al. [23] have discussed the radiative peristaltic flow of Jeffery nanofluid with slip conditions and joule heating.

Few attempts have been devoted to peristaltic flows in presence of heat and mass transfer; such investigations are of great importance, which is due to their extensive applications in medical and bio-engineering sciences, as it may be relevant in many processes in human body like oxygenation in lungs, hemodialysis and nutrients diffuse out of blood. Sohail and nadeem [24] have examined the effects of heat and mass transfer on peristaltic flow of Carreau fluid in a vertical annulus. Eldabe et al. [25] have studied the magneto-hydrodynamic flow and heat transfer for a peristaltic motion of Carreau fluid through a porous medium. Eldabe et al. [26] have studied the peristaltic motion of non-Newtonian fluid 
with heat and mass transfer through a porous medium in channel under uniform magnetic field. Sohail et al. [27] have discussed the effects of heat and mass transfer on peristaltic flow of a nanofluid between eccentric cylinders. Eldabe et al. [28] have discussed the peristaltic pumping of a conduction Sisko fluid through porous medium with heat and mass transfer. Ramesh and Devaker [29] have studied the effects of heat and mass transfer on the peristaltic transport of MHD couple stress fluid through porous medium in a vertical asymmetric channel.

The main aim of this work is to study the peristaltic motion of a Carreau nanofluid with heat and mass transfer through a porous medium in an asymmetric channel under the effects of Hall current, viscous dissipation and Joule heating. The analysis is performed under the well-established long wavelength and low Reynolds number approximations. A detailed mathematical formulation is presented and numerical solution graphically for velocity, temperature, nanoparticle phenomena and pressure gradient have been presented.

\section{Mathematical Formulation of the Problem}

Consider the peristaltic flow of an incompressible viscous electrically conducting nanofluid which obeys Carreau model inside a two dimensional vertical asymmetric channel of width $d_{1}+d_{2}$ through a porous medium. Asymmetry in the channel is produced by propagation of waves along the channel walls traveling with different amplitudes, phases but with constant speed. In the Cartesian coordinates system $(X, Y)$, the right-hand side wall $Y=H_{1}$ and the left-hand side wall $Y=H_{2}$ are given by

$$
\begin{gathered}
Y=H_{1}(X, t)=d_{1}+a_{1} \cos \left(\frac{2 \pi}{\lambda}(X-c t)\right) \\
Y=H_{2}(X, t)=-d_{2}-b_{1} \cos \left(\frac{2 \pi}{\lambda}(X-c t)+\phi\right)
\end{gathered}
$$

where, $a_{1}$ and $b_{1}$ are the amplitudes of right and left walls respectively, $\lambda$ is the wavelength, $t$ is the time, and $\phi$ is the phase which varies in the range $0 \leq \phi \leq \pi$. When $\phi=0$ then symmetric channel with waves out of the phase can be described (i.e. both walls move outward or inward simultaneously), and for $\phi=\pi$, the waves are in phase. Further $d_{1}, d_{2}, a_{1}, b_{1}$ and $\phi$ satisfy the condition:

$$
a_{1}^{2}+b_{1}^{2}+2 a_{1} b_{1} \cos \phi \leq\left(d_{1}+d_{2}\right)^{2},
$$

so that the walls will not intersect with each other.

A strong uniform magnetic field with magnetic flux density $\boldsymbol{B}=\left(0,0, B_{0}\right)$ is applied and the Hall effects are taken into account. The induced magnetic field is neglected by assuming a very small magnetic Reynolds number $\left(R e_{m} \ll<1\right)$, also it is assumed that there is no applied polarization voltage so that the total electric field $\boldsymbol{E}=\mathbf{0}$. The expression for the current density $\boldsymbol{J}$ including the Hall effect and neglecting ion-slip and thermoelectric effects [The generalized Ohm's law] is given by [30]: 


$$
\boldsymbol{J}=\sigma\left[\boldsymbol{E}+\boldsymbol{V} \times \boldsymbol{B}-\frac{1}{\text { en }_{e}}(\boldsymbol{J} \times \boldsymbol{B})\right]
$$

where $\sigma$ is the electrical conductivity of the fluid, $\boldsymbol{V}$ is the velocity vector, $e$ is the electric charge of electrons, $n_{e}$ is the number density of electrons. Equation (1) can be solved in $\boldsymbol{J}$ to yield the Lorentz force vector in the form:

$$
\boldsymbol{J} \times \boldsymbol{B}=\frac{-\sigma B_{0}{ }^{2}}{1+m^{2}}[(U-m V) \hat{i}+(m U+V) \hat{j}]
$$

where $U$ and $V$ are the $X$ and $Y$ components of the velocity vector, $m=\frac{\sigma B_{0}}{e n_{e}}$ is the hall parameter. The heat transfer and nanopatricle processes are maintained by considering temperature $T_{0}, T_{1}$ and nanoparticle phenomena $C_{0}, C_{1}$ to the right and left sides wall, respectively.

The constitutive equation for a carreau fluid is given by [24]:

$$
\begin{gathered}
\frac{\eta-\eta_{\infty}}{\eta_{0}-\eta_{\infty}}=\left[1+(\Gamma \dot{\gamma})^{2}\right]^{\frac{n-1}{2}}, \\
\boldsymbol{\tau}_{i j}=\eta_{0}\left[1+\frac{n-1}{2}(\Gamma \dot{\gamma})^{2}\right] \dot{\gamma}_{i j},
\end{gathered}
$$

in which $\tau_{i j}$ is the extra stress tensor, $\eta_{\infty}$ is the infinite shear rate viscosity, $\eta_{0}$ is the zero shear rate viscosity, $\Gamma$ is the time constant, $n$ is the power law index, and $\dot{\gamma}$ is defined as:

$$
\dot{\gamma}=\sqrt{\frac{1}{2} \sum_{i} \sum_{j} \dot{\gamma}_{i j} \dot{\gamma}_{j i}}=\sqrt{\frac{1}{2} \Pi}
$$

where $\boldsymbol{\Pi}=\operatorname{trac}\left(\operatorname{grad} \boldsymbol{V}+(\operatorname{grad} \boldsymbol{V})^{\mathrm{T}}\right)^{2}$ is the second invariant strain tensor.

The governing equations for the present problem are described as:

Continuity equation

$$
\frac{\partial U}{\partial X}+\frac{\partial V}{\partial Y}=0
$$

Equations of motion

$$
\begin{gathered}
\rho_{f}\left(\frac{\partial U}{\partial t}+U \frac{\partial U}{\partial X}+V \frac{\partial U}{\partial Y}\right) \\
=-\frac{\partial P}{\partial X}+\frac{\partial \tau_{X X}}{\partial X}+\frac{\partial \tau_{X Y}}{\partial Y}-\frac{\eta_{0}}{k_{1}} U+\frac{\sigma B_{0}^{2}}{1+m^{2}}(m V-U)+\rho_{f} g \alpha\left(T-T_{0}\right)++\rho_{f} g \alpha\left(C-C_{0}\right) \\
\rho_{f}\left(\frac{\partial V}{\partial t}+U \frac{\partial V}{\partial X}+V \frac{\partial V}{\partial Y}\right)=-\frac{\partial P}{\partial Y}+\frac{\partial \tau_{Y X}}{\partial X}+\frac{\partial \tau_{Y Y}}{\partial Y}-\frac{\eta_{0}}{k_{1}} V+\frac{\sigma B_{0}{ }^{2}}{1+m^{2}}(m U+V)
\end{gathered}
$$

\section{Energy equation}

$$
\begin{aligned}
& (\rho c)_{f}\left(\frac{\partial T}{\partial t}+U \frac{\partial T}{\partial X}+V \frac{\partial T}{\partial Y}\right) \\
& =K\left(\frac{\partial^{2} T}{\partial X^{2}}+\frac{\partial^{2} T}{\partial Y^{2}}\right)+\left[\begin{array}{c}
\tau_{X X} \frac{\partial U}{\partial X}+\tau_{X Y} \frac{\partial U}{\partial Y} \\
+\tau_{Y X} \frac{\partial V}{\partial X}+\tau_{Y Y} \frac{\partial V}{\partial Y}
\end{array}\right]+\frac{\sigma B_{0}^{2}}{1+m^{2}}\left(U^{2}+V^{2}\right)+(\rho c)_{p}\left\{\begin{array}{c}
D_{B}\left[\frac{\partial C}{\partial X} \frac{\partial T}{\partial X}+\frac{\partial C}{\partial Y} \frac{\partial T}{\partial Y}\right. \\
+\frac{D_{T}}{T_{0}}\left[\left(\frac{\partial T}{\partial X}\right)^{2}+\left(\frac{\partial T}{\partial Y}\right)^{2}\right.
\end{array}\right]
\end{aligned}
$$




\section{Concentration equation}

$$
\frac{\partial C}{\partial t}+U \frac{\partial C}{\partial X}+V \frac{\partial C}{\partial Y}=D_{B}\left(\frac{\partial^{2} C}{\partial X^{2}}+\frac{\partial^{2} C}{\partial Y^{2}}\right)+\frac{D_{T}}{T_{0}}\left(\frac{\partial^{2} T}{\partial X^{2}}+\frac{\partial^{2} T}{\partial Y^{2}}\right)
$$

where $\rho_{f}$ is the density of the fluid, $P$ is the pressure, $k_{1}$ is the permeability of the porous medium, $g$ is the acceleration due to gravity, $\alpha$ is the volume expansion coefficient, $T$ is the temperature of the fluid, $C$ is the nanoprticle concentration, $(\rho c)_{f}$ is the heat capacity of the fluid, $K$ is the thermal conductivity, $(\rho c)_{p}$ is the effective heat capacity of the nanoparticle material, $D_{B}$ is the Brownian diffusion coefficient and $D_{T}$ is the thermophoretic diffusion.

Introducing a wave frame $(x, y)$ moving with the velocity $c$ away from the fixed frame $(X, Y)$ by the transformation

$$
x=X-c t, y=Y, u=U-c, p(x)=P(X, t)
$$

in which $(x, y),(u, v)$ and $P$ are the coordinates, velocity components and pressure in the wave frame.

Defining the following non-dimensional quantities

$$
\begin{aligned}
& \bar{x}=\frac{x}{\lambda}, \bar{y}=\frac{y}{d_{1}}, \bar{u}=\frac{u}{c}, \bar{v}=\frac{v}{c}, \bar{t}=\frac{t}{\lambda}, \delta=\frac{d_{1}}{\lambda}, d=\frac{d_{2}}{d_{1}}, a=\frac{a_{1}}{d_{1}}, \\
& b=\frac{b_{1}}{d_{1}}, h_{1}=\frac{H_{1}}{d_{1}}, h_{2}=\frac{H_{2}}{d_{2}}, \bar{p}=\frac{d_{1}^{2} p}{\eta_{0} c \lambda}, \operatorname{Re}=\frac{\rho_{f} c d_{1}}{\eta_{0}}, \bar{\psi}=\frac{\psi}{c d_{1}}, \\
& \bar{\tau}_{\overline{x x}}=\frac{\lambda}{\eta_{0} c} \tau_{x x}, \bar{\tau}_{\overline{x y}}=\frac{d_{1}}{\eta_{0} c} \tau_{x y}, \bar{\tau}_{\overline{y y}}=\frac{d_{1}}{\eta_{0} c} \tau_{y y}, W e=\frac{\Gamma c}{d_{1}}, \bar{\gamma}=\frac{d_{1} \dot{\gamma}}{c}, \\
& s=\frac{k_{1}}{d_{1}^{2}}, M=\sqrt{\frac{\sigma}{\eta_{0}}} B_{0} d_{1}, \bar{\theta}=\frac{T-T_{0}}{T_{1}-T_{0}}, \bar{\Omega}=\frac{C-C_{0}}{C_{1}-C_{0}}, \operatorname{Pr}=\frac{\mu}{\rho_{f} \alpha}, \\
& G r=\frac{\rho_{f} g \alpha d_{1}^{2}\left(T_{1}-T_{0}\right)}{c \eta_{0}}, B r=\frac{\rho_{f} g \alpha d_{1}^{2}\left(C_{1}-C_{0}\right)}{c \eta_{0}}, E_{c}=\frac{c_{1}^{2}}{c_{f}\left(T_{1}-T_{0}\right)}, \\
& S_{c}=\frac{\mu}{\rho_{f} D_{B}}, N_{b}=\frac{\tau D_{B}\left(C_{1}-C_{0}\right)}{\alpha}, N_{t}=\frac{\tau D_{B}\left(T_{1}-T_{0}\right)}{\alpha}, \\
& \alpha=\frac{K}{(\rho c)_{f}}, \tau=\frac{(\rho c)_{p}}{(\rho c)_{f}} .
\end{aligned}
$$

where $\delta$ is the dimensionless wave number, $R e$ is the Reynolds number, $W e$ is the Weissenberg number, $S$ is the porosity parameter, $M$ is the magnetic parameter, $\mathrm{Gr}$ is the local temperature Grashof number, $\mathrm{Br}$ is the local nanoparticle Grashof number, $\operatorname{Pr}$ is the Prandtl number, $E_{c}$ is the Eckert number, $S_{c}$ is the Schmidt number, $N b$ is the Brownain motion parameter and $N_{t}$ is the thermophoresis parameter.

Using Equation (14) and the above set of non-dimensional quantities (15) into Equations (10)-(13), the resulting equations in terms of stream function $\psi\left(u=\frac{\partial \psi}{\partial x}, v=-\delta \frac{\partial \psi}{\partial y}\right)$ can be written after dropping bars in the following

Non-dimensionless form as: 


$$
\begin{aligned}
& \operatorname{Re} \delta\left[\left(\frac{\partial \psi}{\partial y} \frac{\partial}{\partial x}-\frac{\partial \psi}{\partial x} \frac{\partial}{\partial y}\right) \frac{\partial \psi}{\partial y}\right] \\
& =-\frac{\partial p}{\partial x}+\delta^{2} \frac{\partial}{\partial x}\left(\tau_{x x}\right)+\frac{\partial}{\partial y}\left(\tau_{x y}\right)-\delta \frac{m M^{2}}{1+m^{2}}\left(\frac{\partial \psi}{\partial x}\right)-\frac{M^{2}}{1+m^{2}}\left(\frac{\partial \psi}{\partial y}+1\right)-\frac{1}{s}\left(\frac{\partial \psi}{\partial y}+1\right)+G r \theta+B r \Omega \\
& \operatorname{Re} \delta^{3}\left[\left(-\frac{\partial \psi}{\partial y} \frac{\partial}{\partial x}+\frac{\partial \psi}{\partial x} \frac{\partial}{\partial y}\right) \frac{\partial \psi}{\partial x}\right] \\
& =-\frac{\partial p}{\partial y}+\delta^{2} \frac{\partial}{\partial x}\left(\tau_{y x}\right)+\delta \frac{\partial}{\partial y}\left(\tau_{y y}\right)-\delta \frac{m M^{2}}{1+m^{2}}\left(\frac{\partial \psi}{\partial y}-1\right)-\delta^{2} \frac{M^{2}}{1+m^{2}}\left(\frac{\partial \psi}{\partial y}+1\right)-\delta \frac{1}{s}\left(\frac{\partial \psi}{\partial x}\right) \\
& \left.\operatorname{Re} \delta\left[\frac{\partial \psi}{\partial y} \frac{\partial \theta}{\partial x}-\frac{\partial \psi}{\partial x} \frac{\partial \theta}{\partial y}\right]=\frac{1}{P_{r}}\left(\frac{\partial^{2} \theta}{\partial y^{2}}+\delta^{2} \frac{\partial^{2} \theta}{\partial x^{2}}\right)+E_{c}\left[\begin{array}{c}
\delta^{2} \tau_{x x} \frac{\partial^{2} \psi}{\partial x \partial y}+\tau_{x y} \frac{\partial^{2} \psi}{\partial y^{2}} \\
-\delta^{2} \tau_{y x} \frac{\partial^{2} \psi}{\partial x^{2}}-\delta \tau_{y y} \frac{\partial^{2} \psi}{\partial y \partial x}
\end{array}\right)\right] \\
& +E_{c} \frac{M^{2}}{1+m^{2}}\left[\begin{array}{l}
\delta^{2}\left(\frac{\partial \psi}{\partial x}\right)^{2}+ \\
\left(\frac{\partial \psi}{\partial y}+1\right)^{2}
\end{array}\right] E_{c} \frac{M^{2}}{1+m^{2}}\left[\begin{array}{l}
\delta^{2}\left(\frac{\partial \psi}{\partial x}\right)^{2}+ \\
\left(\frac{\partial \psi}{\partial y}+1\right)^{2}
\end{array}\right]+\frac{N_{t}}{\operatorname{Pr}}\left[\delta^{2}\left(\frac{\partial \theta}{\partial x}\right)^{2}+\left(\frac{\partial \theta}{\partial y}\right)^{2}\right] \\
& \operatorname{Re} \delta S_{c}\left[\frac{\partial \psi}{\partial y} \frac{\partial \Omega}{\partial x}-\frac{\partial \psi}{\partial x} \frac{\partial \Omega}{\partial y}\right]=\left(\frac{\partial^{2} \Omega}{\partial y^{2}}+\delta^{2} \frac{\partial^{2} \Omega}{\partial x^{2}}\right)+\frac{N_{t}}{N_{b}}\left(\frac{\partial^{2} \theta}{\partial y^{2}}+\delta^{2} \frac{\partial^{2} \theta}{\partial x^{2}}\right) \\
& \tau_{x x}=2\left[1+\frac{n-1}{2} W e^{2} \dot{\gamma}^{2}\right] \frac{\partial^{2} \psi}{\partial x \partial y}, \\
& \tau_{x y}=\left[1+\frac{n-1}{2} W e^{2} \dot{\gamma}^{2}\right]\left(\frac{\partial^{2} \psi}{\partial y^{2}}-\delta^{2} \frac{\partial^{2} \psi}{\partial x^{2}}\right)=\tau_{y x}, \\
& \tau_{y y}=2 \delta\left[1+\frac{n-1}{2} W e^{2} \dot{\gamma}^{2}\right] \frac{\partial^{2} \psi}{\partial x \partial y},
\end{aligned}
$$

and,

$$
\dot{\gamma}=\left[2 \delta^{2}\left(\frac{\partial^{2} \psi}{\partial x \partial y}\right)^{2}+\left(\frac{\partial^{2} \psi}{\partial y^{2}}-\delta^{2} \frac{\partial^{2} \psi}{\partial x^{2}}\right)^{2}+2 \delta^{2}\left(\frac{\partial^{2} \psi}{\partial y \partial x}\right)^{2}\right]^{\frac{1}{2}}
$$

Under the assumption of long wavelength $\delta \ll 1$ and low Reynolds number, Equations (16)-(19) become

$$
\begin{array}{r}
0=-\frac{\partial p}{\partial x}+\frac{\partial}{\partial y}\left[1+\frac{n-1}{2} W e^{2}\left(\frac{\partial^{2} \psi}{\partial y^{2}}\right)^{2}\right] \frac{\partial^{2} \psi}{\partial y^{2}}-\left(\frac{M^{2}}{1+m^{2}}+\frac{1}{s}\right)\left[\frac{\partial \psi}{\partial y}+1\right]+G r \theta+B r \Omega( \\
0=-\frac{\partial p}{\partial y} \\
0=\frac{1}{\operatorname{Pr}} \frac{\partial^{2} \theta}{\partial y^{2}}+E_{c}\left[\left(\frac{\partial^{2} \psi}{\partial y^{2}}\right)^{2}+\frac{n-1}{2} W e^{2}\left(\frac{\partial^{2} \psi}{\partial y^{2}}\right)^{4}\right]+E_{c} \frac{M^{2}}{1+m^{2}}\left(\frac{\partial \psi}{\partial y}+1\right)^{2}+\frac{N_{b}}{\operatorname{Pr}}\left(\frac{\partial \Omega}{\partial y} \frac{\partial \theta}{\partial y}\right)+\frac{N_{t}}{\operatorname{Pr}}\left(\frac{\partial \theta}{\partial y}\right)^{2}
\end{array}
$$




$$
0=\frac{\partial^{2} \Omega}{\partial y^{2}}+\frac{N_{t}}{N_{b}} \frac{\partial^{2} \theta}{\partial y^{2}}
$$

Eliminating the pressure from Equations (24) and (25) yields

$$
\frac{\partial^{2}}{\partial y^{2}}\left[\left(1+\frac{n-1}{2} W e^{2}\left(\frac{\partial^{2} \psi}{\partial y^{2}}\right)^{2}\right) \frac{\partial^{2} \psi}{\partial y^{2}}-\left(\frac{M^{2}}{1+m^{2}}+\frac{1}{s}\right) \psi\right]+G r \frac{\partial \theta}{\partial y}+B r \frac{\partial \Omega}{\partial y}=0
$$

The non-dimensional boundaries will take the form

$$
h_{1}=1+a \cos (2 \pi x), \quad h_{2}=-d-b \cos (2 \pi x+\phi)
$$

where $a, b, \phi, d$ satisfies the relation

$$
a^{2}+b^{2}+2 a b \cos \phi \leq(1+d)^{2}
$$

The corresponding boundary conditions are

$$
\begin{aligned}
& \psi=\frac{F}{2}, \frac{\partial \psi}{\partial y}=-1, \theta=0, \Omega=0, \text { at } y=h_{1} \\
& \psi=-\frac{F}{2}, \frac{\partial \psi}{\partial y}=-1, \theta=1, \Omega=1, \text { at } y=h_{2}
\end{aligned}
$$

where $F$ is the dimensionless average flux in the wave frame defined by

$$
F=\int_{h_{2}(x)}^{h_{1}(x)} \frac{\partial \psi}{\partial y} \mathrm{~d} y
$$

The time mean $Q$ in the wave frame is defined by

$$
Q=F+1+d
$$

\section{Results and Discussion}

In this work, a program was designed by Mathematica Software (version 10) simulate the application of parametric ND Solve package to find the numerical behaviors of our dimensionless system. Graphical results to the velocity $u$, the temperature $\theta$, the nano particle concentration $\Omega$ and the pressure gradient $\frac{\mathrm{d} p}{\mathrm{~d} x}$ are obtained under the impact of emerging parameters at moving boundaries of the fluid.

\subsection{Velocity Profile}

Figures 1-5 represent the impact of the Weissenberg number we, the power law index $n$, the magnetic parameter $M$, the thermophoresis parameter $n_{t}$ and flow rate $Q$ on the velocity profile. Figure 1 and Figure 2 depict that the behaviour of the velocity near the channel walls and at the center is not similar in view of we and $n$. The velocity field increases with the increase of we and $n$ near the channel walls, however it decreases at the center of the channel. Figure 3 and Figure 4 show that the effect of $M$ and $n_{t}$ on the velocity profile is the same and opposite to that of we and $n$, as by increasing $M$ and $n_{t}$ the velocity decreases near the channel walls and increases at the center of the channel, but a reduction in $u$ occurs and variation in $u$ becomes narrow in some sections of the 

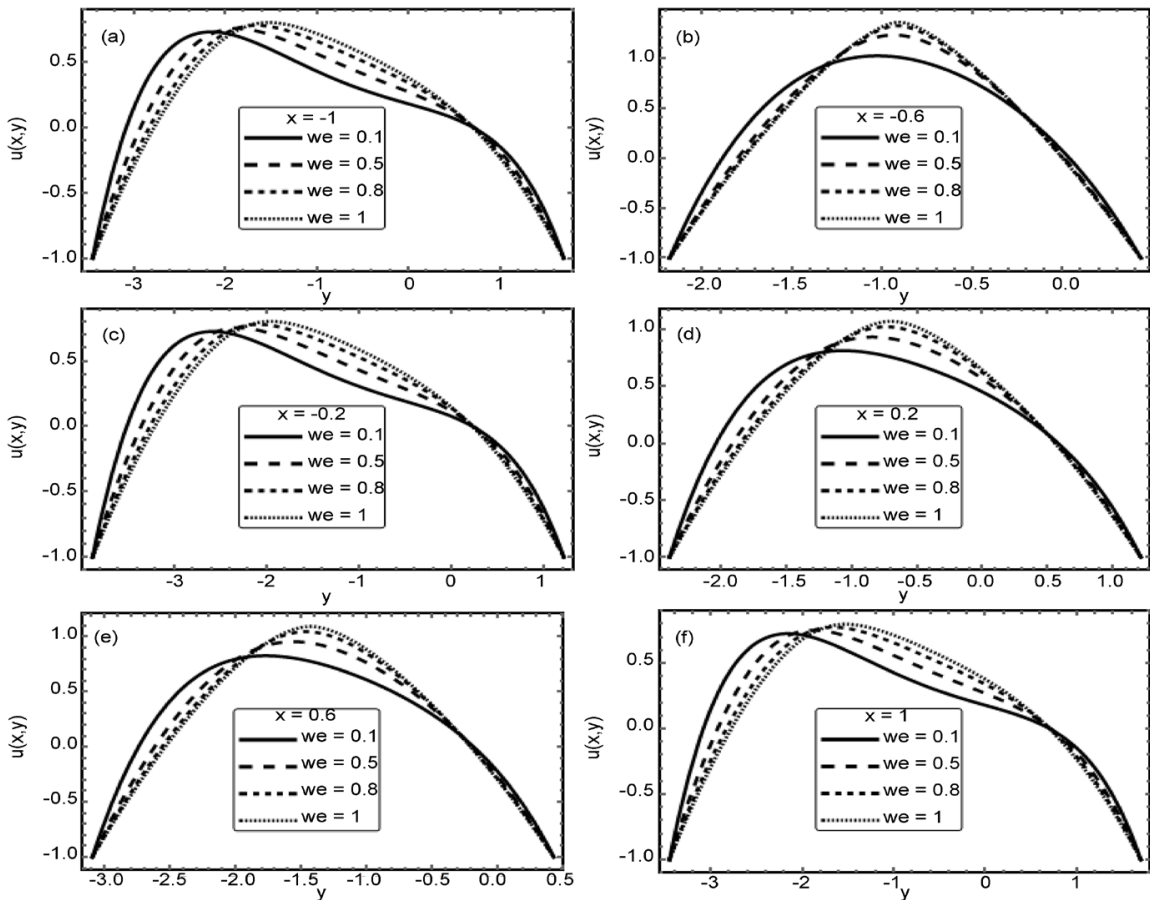

Figure 1. The velocity profile $\mathrm{u}$ is plotted against $\mathrm{y}$ for several values of $W e$ at $a=0.7$, $b=0.9, d=3, \phi=\frac{\pi}{3}, \quad \operatorname{Pr}=3, \quad E c=0.2, \quad m=1, \quad M=1.2, \quad N_{t}=0.7, \quad N b=0.7$, $n=3, G r=1, B r=2, s=2, F=1$, when (a) $x=-1$, (b) $x=-0.6$, (c) $x=-0.2$, (d) $x=$ 0.2 , (e) $x=0.6$, (f) $x=1$.
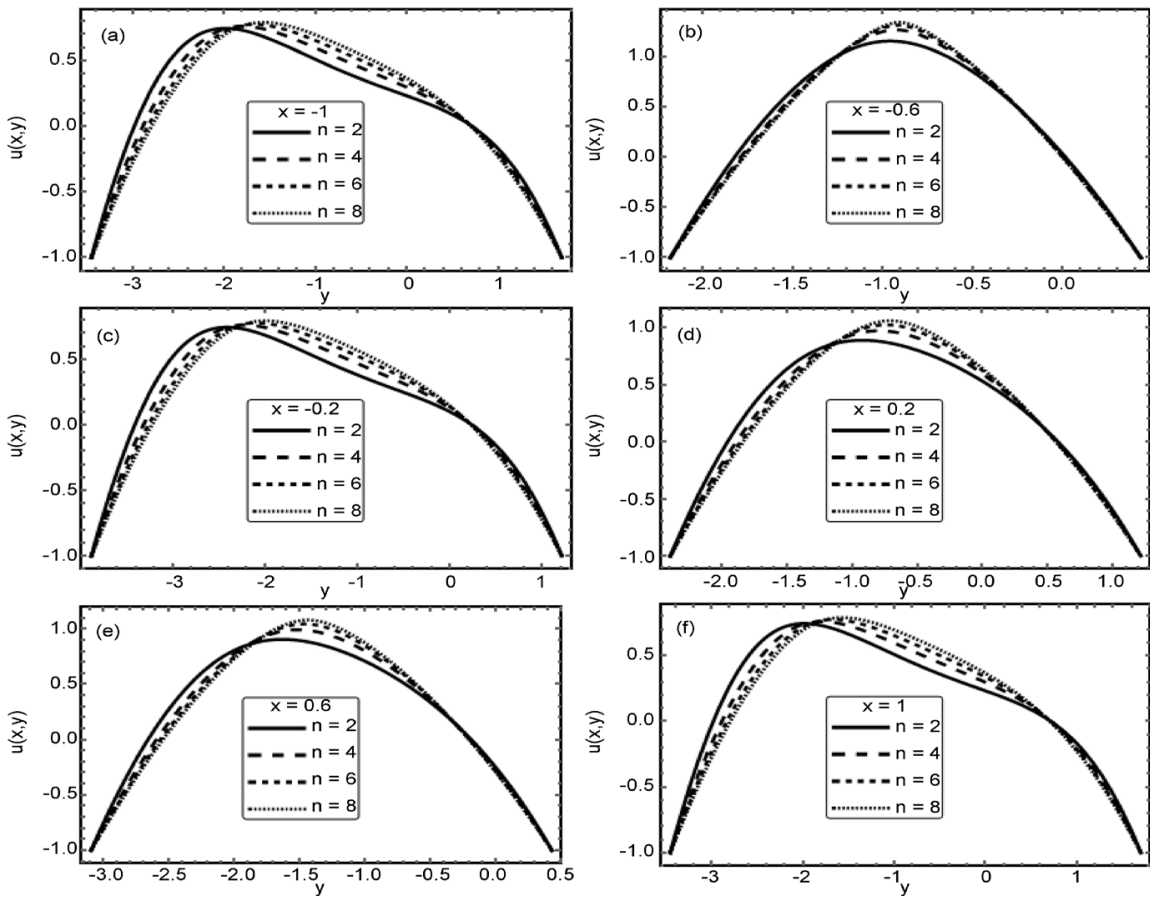

Figure 2. The velocity profile $u$ is plotted against y for several values of $n$ at $a=0.7$, $b=0.9, d=3, \phi=\frac{\pi}{3}, \operatorname{Pr}=3, E c=0.2, W e=0.5, m=1, M=1.2, N_{t}=0.7$, $\mathrm{Nb}=0.7, \mathrm{Gr}=1, \mathrm{Br}=2, \mathrm{~s}=2, F=1$, when (a) $x=-1$, (b) $x=-0.6$, (c) $x=-0.2$, (d) $x=0.2$, (e) $x=0.6$, (f) $x=1$. 

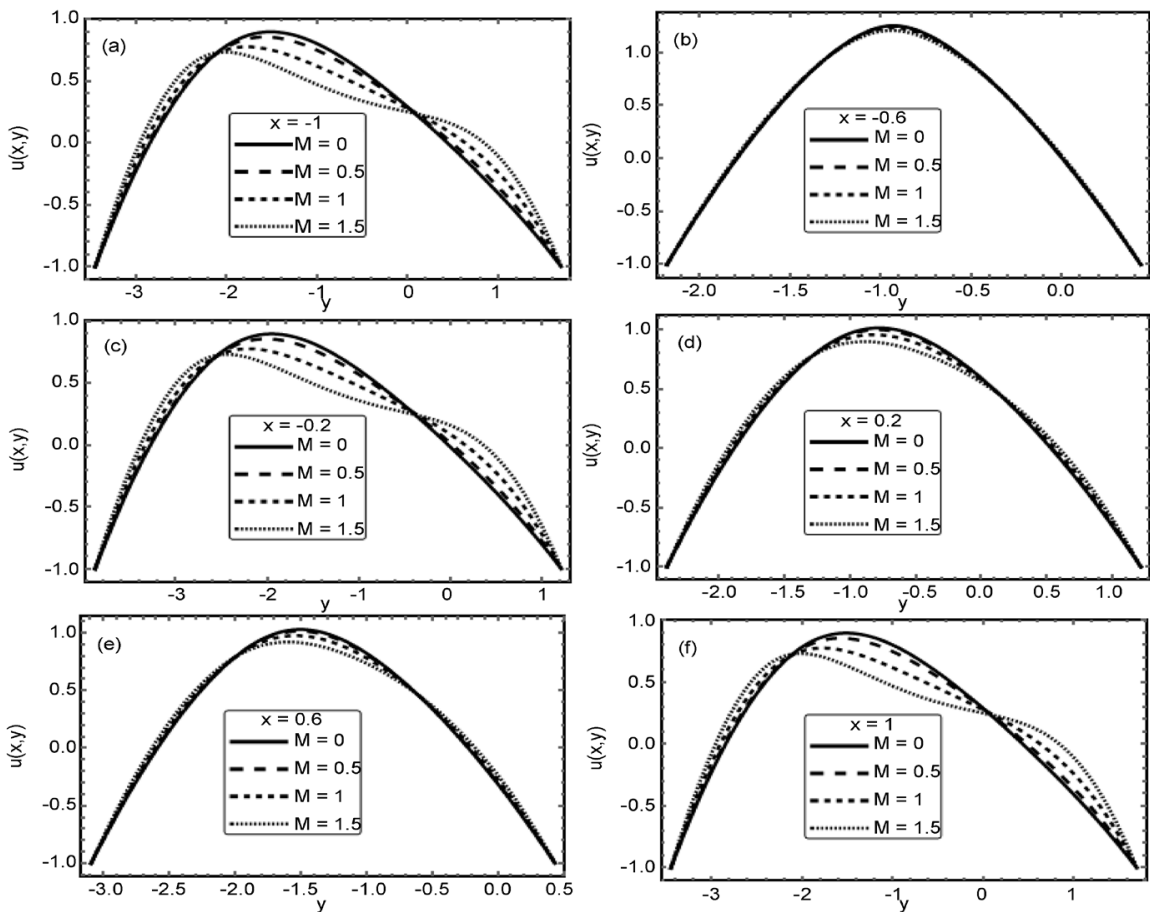

Figure 3. The velocity profile $u$ is plotted against y for several values of $M$ at $a=0.7$, $b=0.9, d=3, \phi=\frac{\pi}{3}, \operatorname{Pr}=3, E c=0.2, W e=0.5, m=1, N_{t}=0.7, N b=0.7$, $n=3, G r=1, B r=2, s=2, F=1$, when (a) $x=-1$, (b) $x=-0.6$, (c) $x=-0.2$, (d) $x=$ 0.2 , (e) $x=0.6$, (f) $x=1$.
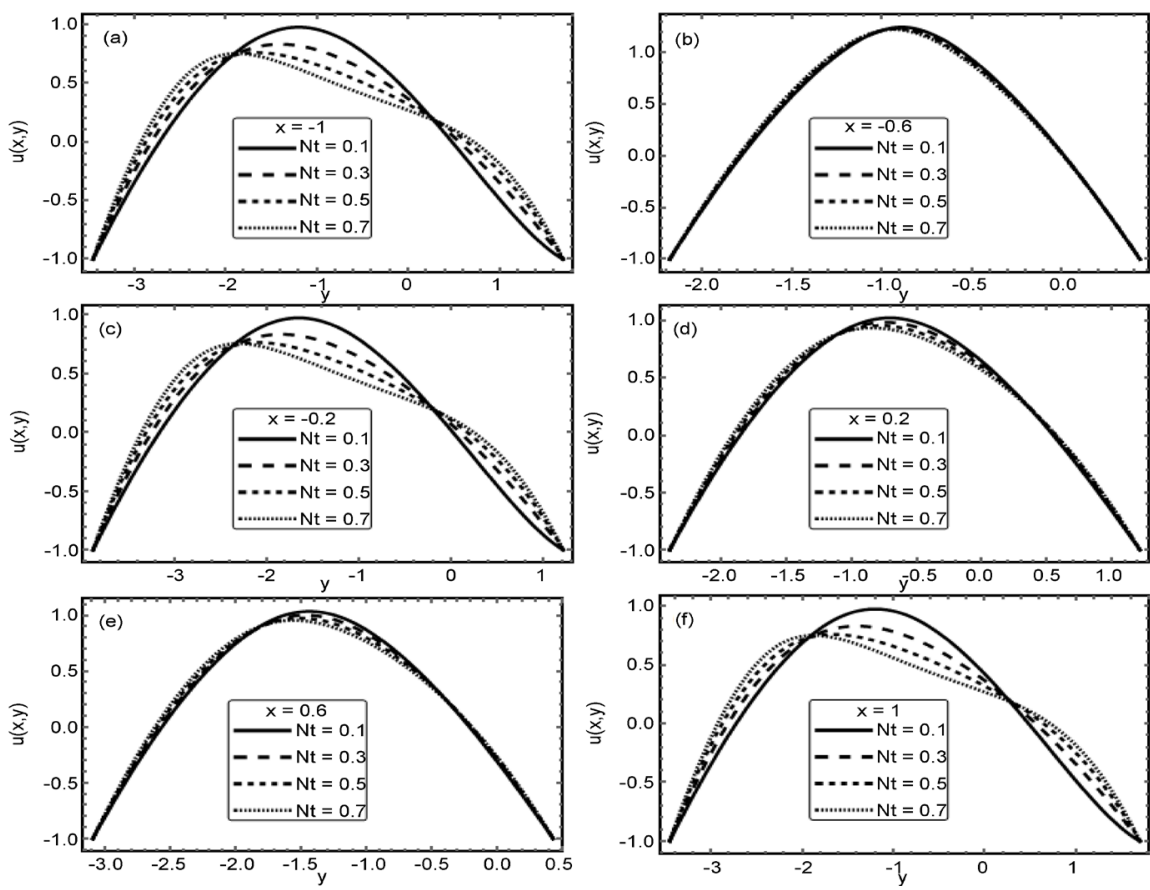

Figure 4. The velocity profile $u$ is plotted against y for several values of $N t$ at $a=0.7$, $b=0.9, d=3, \phi=\frac{\pi}{3}, \operatorname{Pr}=3, E c=0.2, W e=0.5, \quad m=1, \quad M=1.2, N b=0.7$, $n=3, G r=1, B r=2, s=2, F=1$, when (a) $x=-1$, (b) $x=-0.6$, (c) $x=-0.2$, (d) $x=$ 0.2 , (e) $x=0.6$, (f) $x=1$. 

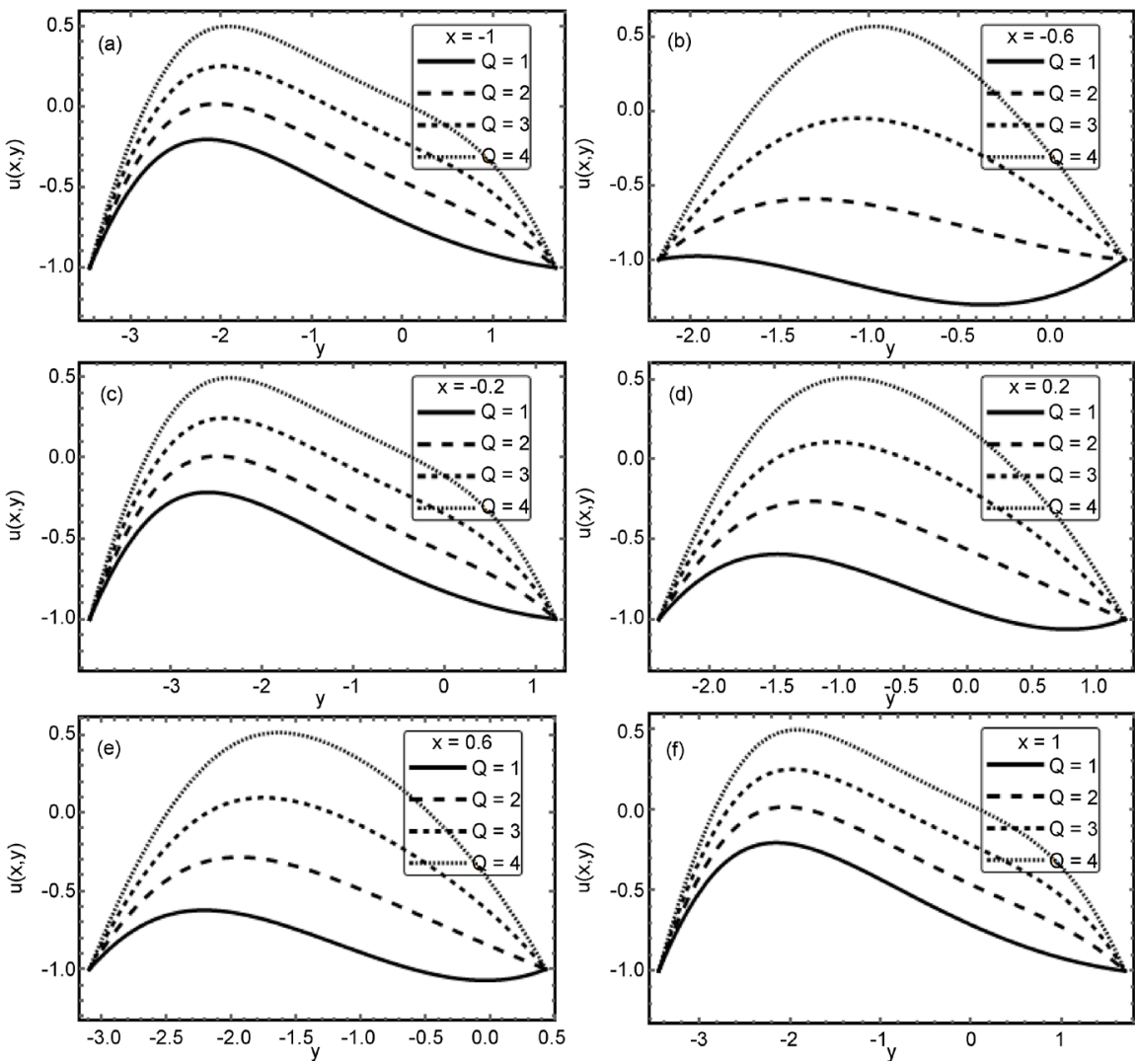

Figure 5. The velocity profile $u$ is plotted against y for several values of $Q$ for $a=0.7$, $b=0.9, d=3, \phi=\frac{\pi}{3}, \operatorname{Pr}=3, E c=0.2, W e=0.5, \quad m=1, M=1.2, N_{t}=0.7$, $N b=0.7, n=3, G r=1, B r=2, s=2$, when (a) $x=-1$, (b) $x=-0.6$, (c) $x=-0.2$, (d) $x=0.2$, (e) $x=0.6$, (f) $x=1$.

fluid motion see (Figure 3(b), Figure 3(d) and Figure 3(e)), and Figure 4(b), Figure 4(d) and Figure 4(e). It is noticed through Figure 5 that the velocity profile increases with increasing $Q$.

\subsection{Temperature Profile}

The variation of temperature profile for different values of the magnetic parameter $M$, the Brownain motion parameter $N b$, the Prandtl number $P r$ the local nanoparticle Grashof number $b r$ and the thermophoresis parameter $n_{t}$ are plotted in Figures 6-10. It is clear that the temperature profile increases when there is an increase in $M, N b$ and $P r$, however it decreases with the increase in $b r$ and $n_{t}$.

\subsection{Concentration Profile}

Figures 11-15 describe the variation of the concentration profile for several values of the magnetic parameter $M$, the prandtl number $P r$, the thermophoresis parameter $n_{t}$ the Brownain motion parameter $N b$ and the local nanoparticle Grashof number $b r$. From Figures 11-13 it is clear that by increasing $M, P r$, and $n_{b}$ the concentration profile desreases, while from Figure 14 and Figure 15 we observe that the concentration profile increases with the increase in $N b$ and $n_{t}$. 

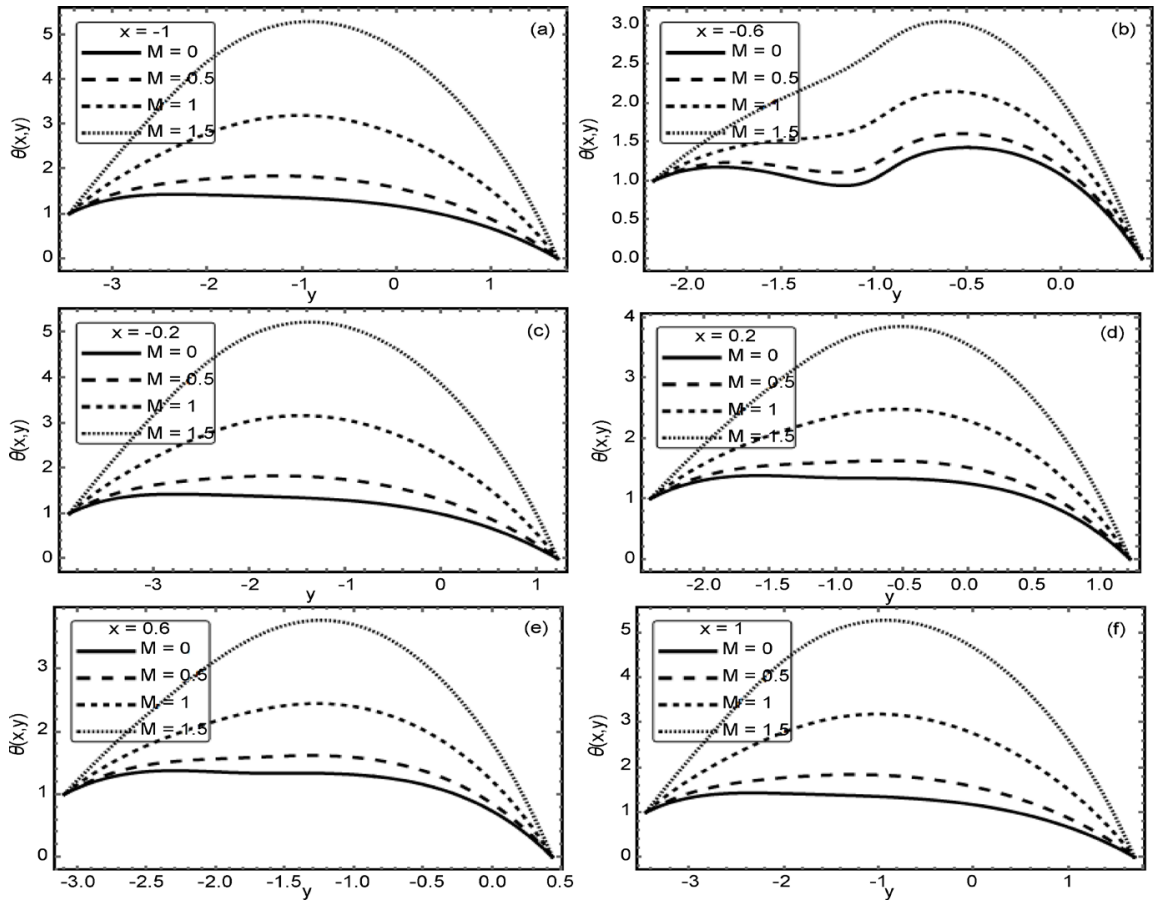

Figure 6. The temperature profile $\theta$ is plotted against y for several values of $M$ at $a=0.7, b=0.9, d=3, \phi=\frac{\pi}{3}, \quad \operatorname{Pr}=3, E c=0.2, \quad W e=0.5, \quad m=1, N_{t}=0.7$, $N b=0.7, n=3, G r=1, B r=2, s=2, F=1$, when (a) $x=-1$, (b) $x=-0.6$, (c) $x=$ -0.2 , (d) $x=0.2$, (e) $x=0.6$, (f) $x=1$.
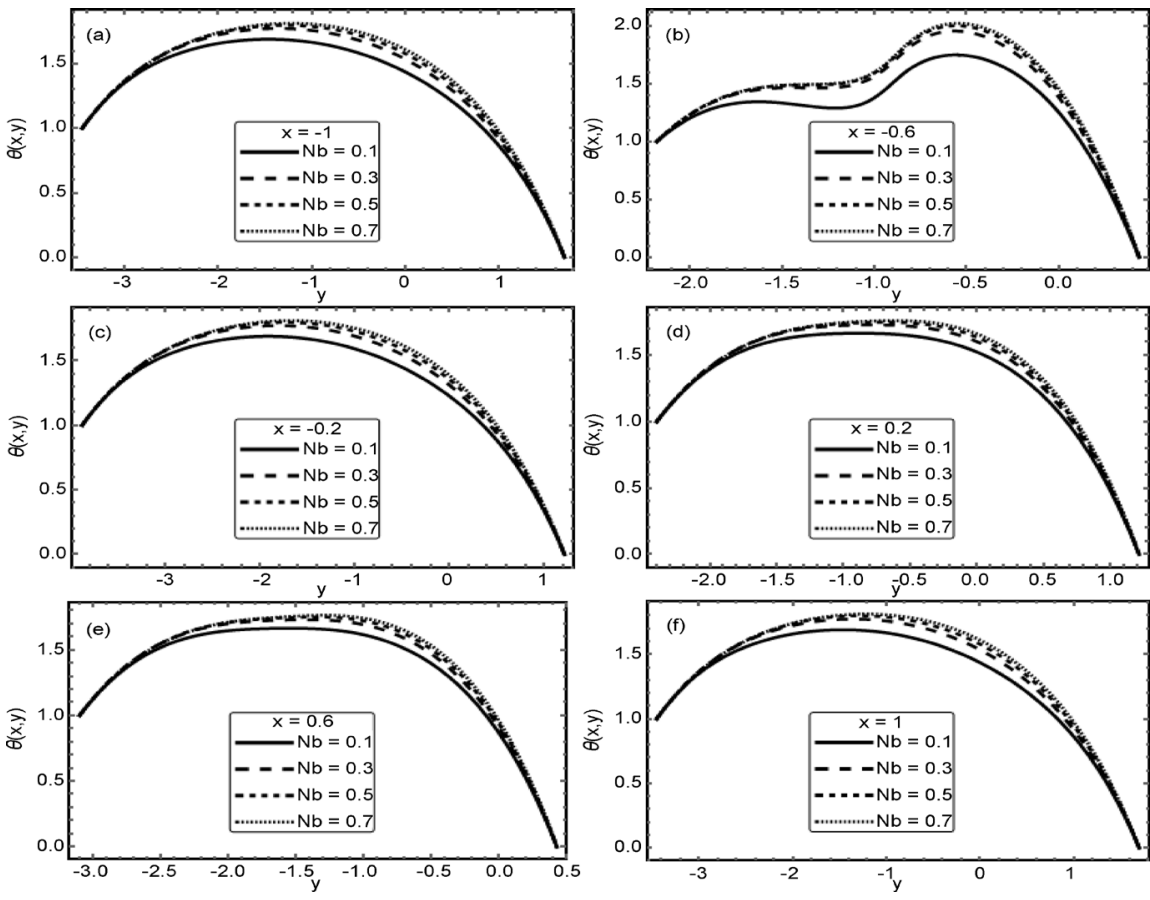

Figure 7. The temperature profile $\theta$ is plotted against y for several values of $N b$ at $a=0.7, b=0.9, d=3, \phi=\frac{\pi}{3}, \operatorname{Pr}=3, \quad E c=0.2, \quad W e=0.5, \quad m=1, \quad M=0.5$, $N_{t}=0.7, n=3, G r=1, B r=2, s=2, F=1$, when (a) $x=-1$, (b) $x=-0.6$, (c) $x=$ -0.2 , (d) $x=0.2$, (e) $x=0.6$, (f) $x=1$. 

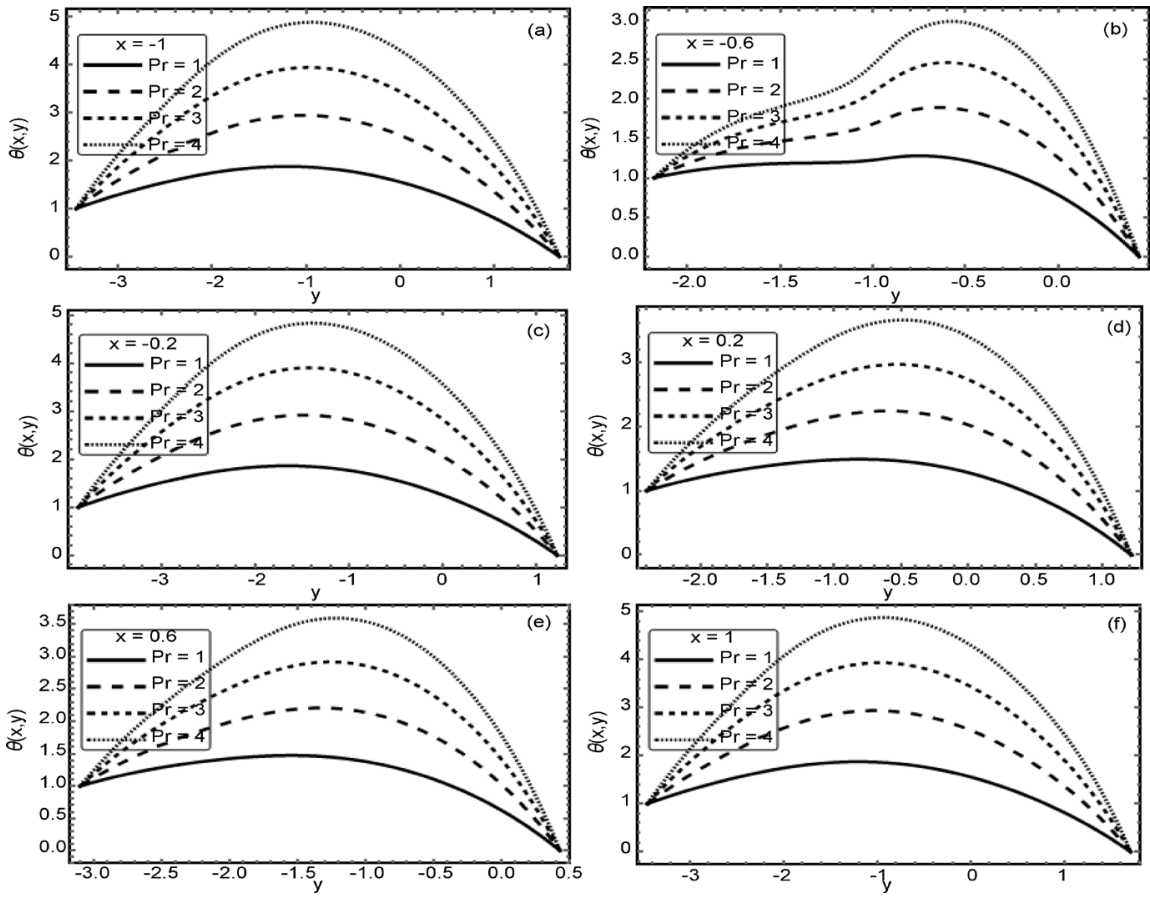

Figure 8. The temperature profile $\theta$ isplotted against y for several values of $\operatorname{Pr}$ at $a=0.7, b=0.9, d=3, \phi=\frac{\pi}{3}, E c=0.2, W e=0.5, m=1, M=0.5, N_{t}=0.7$, $N b=0.7, n=3, G r=1, B r=2, s=2, F=1$, when (a) $x=-1$, (b) $x=-0.6$, (c) $x=$ -0.2 , (d) $x=0.2$, (e) $x=0.6$, (f) $x=1$.
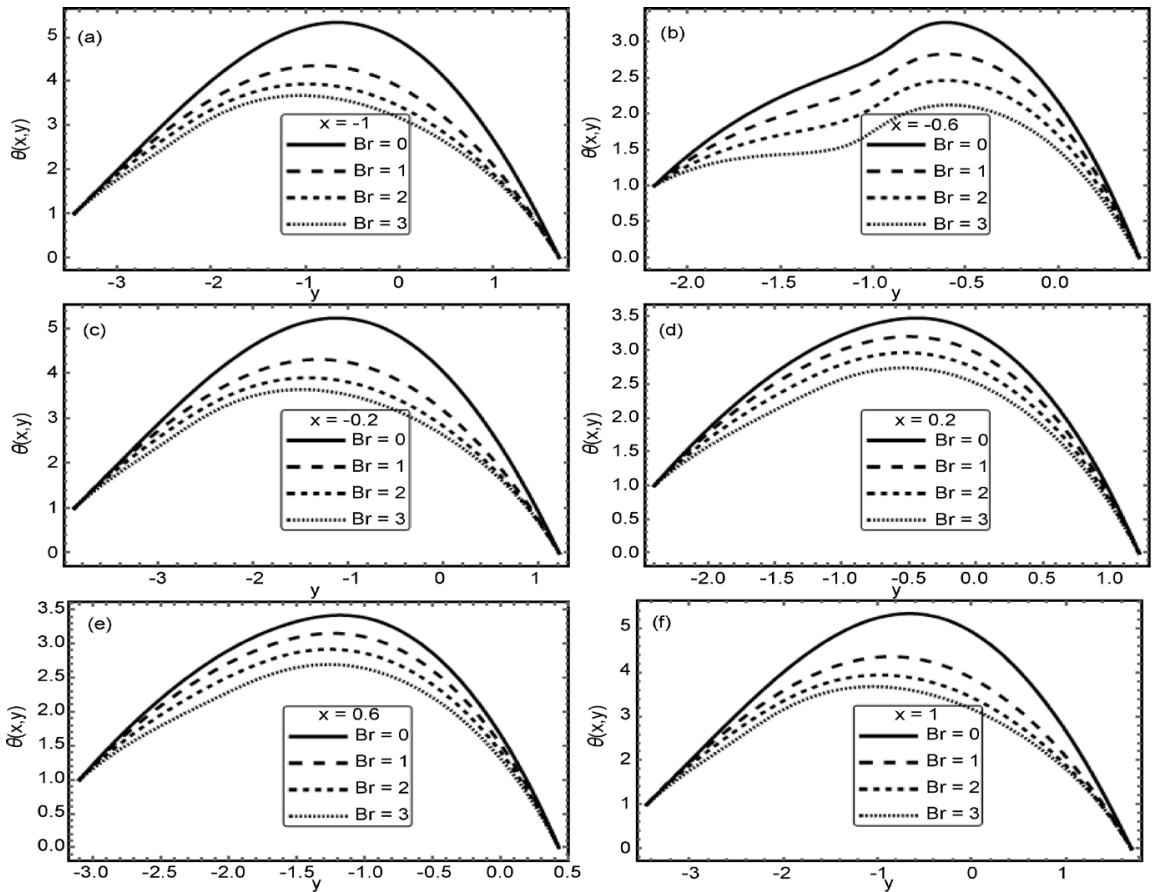

Figure 9. The temperature profile $\theta$ is plotted against y for several values of $B r$ at $a=0.7, b=0.9, d=3, \phi=\frac{\pi}{3}, \operatorname{Pr}=3, \quad E c=0.2, \quad W e=0.5, \quad m=1, \quad M=1.2$, $N_{t}=0.7, N b=0.7, n=3, G r=1, s=2, F=1$, when (a) $x=-1$, (b) $x=-0.6$, (c) $x=$ -0.2 , (d) $x=0.2$, (e) $x=0.6$, (f) $x=1$. 

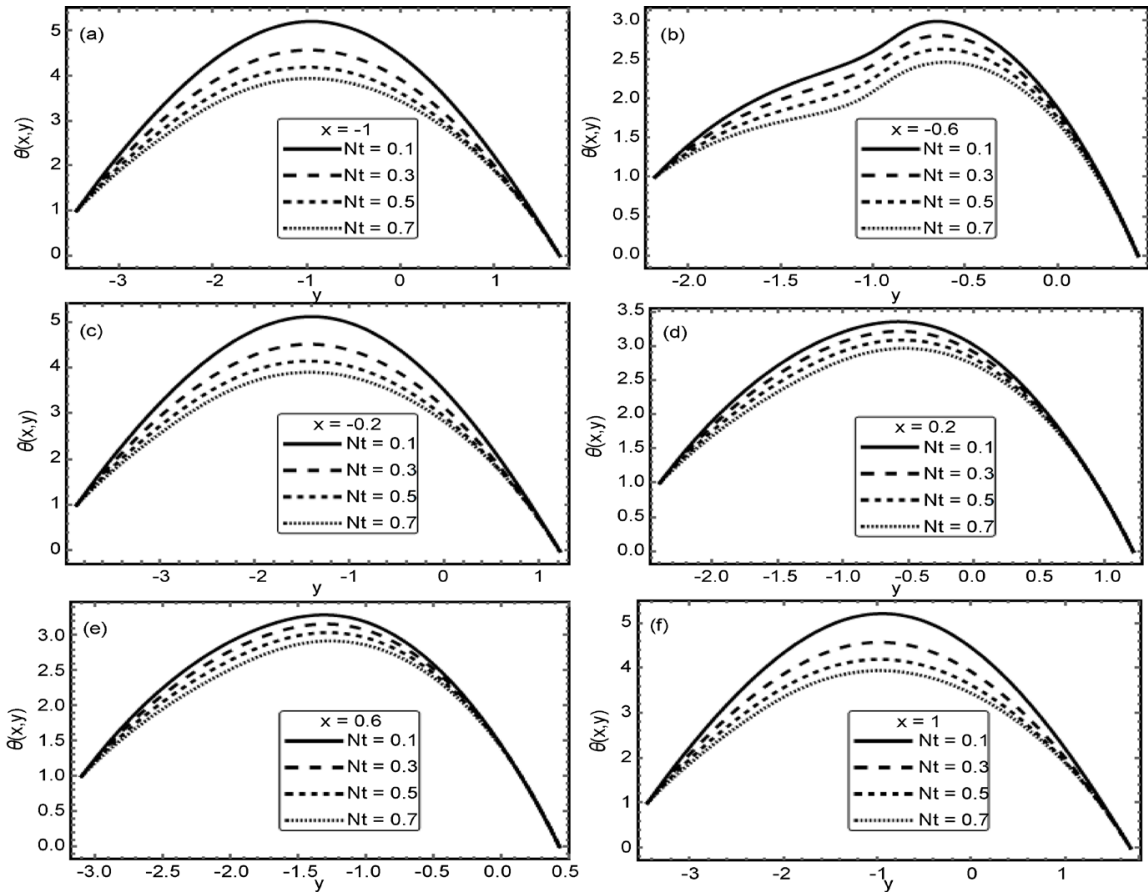

Figure 10. The temperature profile $\theta$ is plotted against y for several values of $N t$ at $a=0.7, b=0.9, d=3, \phi=\frac{\pi}{3}, \operatorname{Pr}=3, E c=0.2, \quad W e=0.5, \quad m=1, \quad M=1.2$, $N b=0.7, n=3, G r=1, B r=2, s=2, F=1$, when (a) $x=-1$, (b) $x=-0.6$, (c) $x=$ -0.2 , (d) $x=0.2$, (e) $x=0.6$, (f) $x=1$.
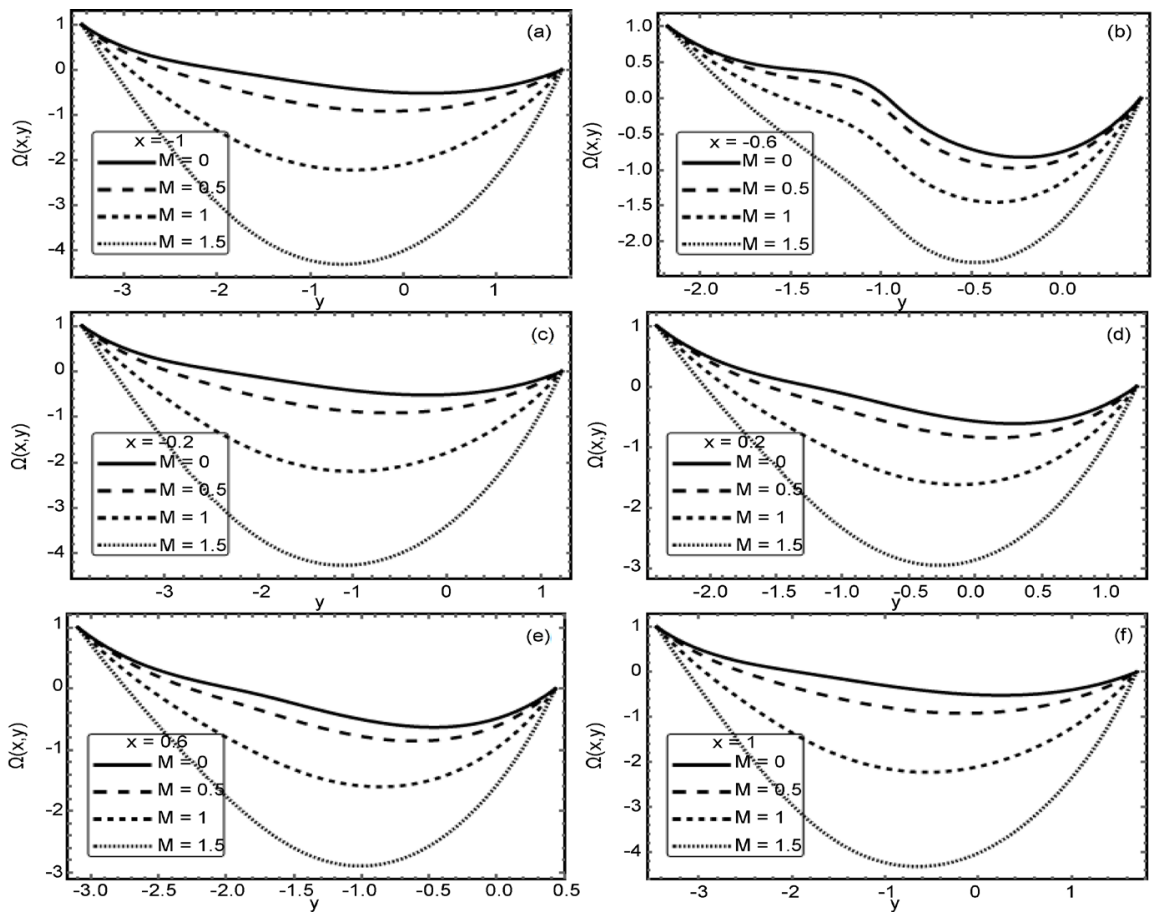

Figure 11. The concentration profile $\Omega$ is plotted against y for several values of $M$ at $a=0.7, b=0.9, d=3, \phi=\frac{\pi}{3}, \operatorname{Pr}=3, E c=0.2, \quad W e=0.5, \quad m=1, N_{t}=0.7$, $N b=0.7, n=3, G r=1, B r=2, s=2, F=1$, when (a) $x=-1$, (b) $x=-0.6$, (c) $x=$ -0.2 , (d) $x=0.2$, (e) $x=0.6$, (f) $x=1$. 

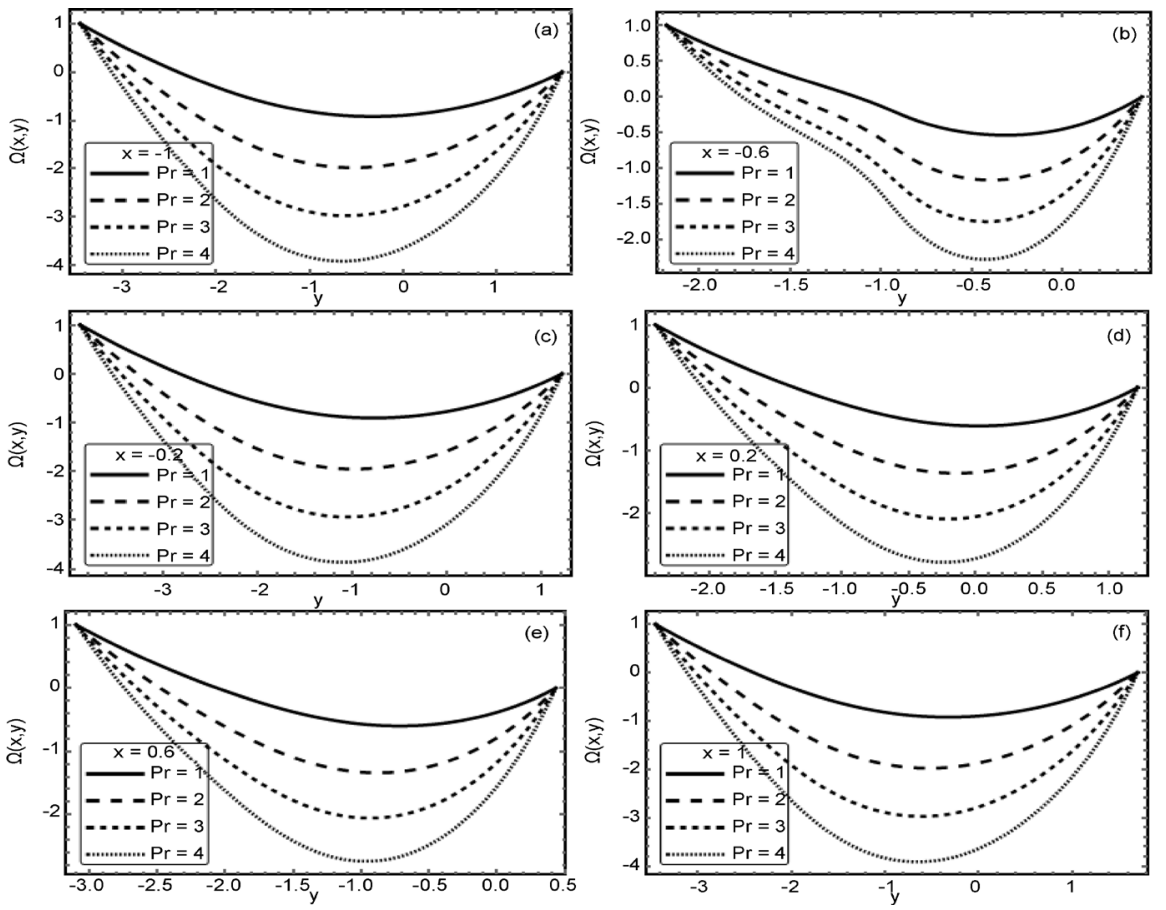

Figure 12. The concentration profile $\Omega$ is plotted against y for several values of $\operatorname{Pr}$ at $a=0.7, b=0.9, d=3, \phi=\frac{\pi}{3}, E c=0.2, W e=0.5, m=1, N_{t}=0.7, \quad M=1.2$, $N b=0.7, n=3, G r=1, B r=2, s=2, F=1$, when (a) $x=-1$, (b) $x=-0.6$, (c) $x=$ -0.2 , (d) $x=0.2$, (e) $x=0.6$, (f) $x=1$.
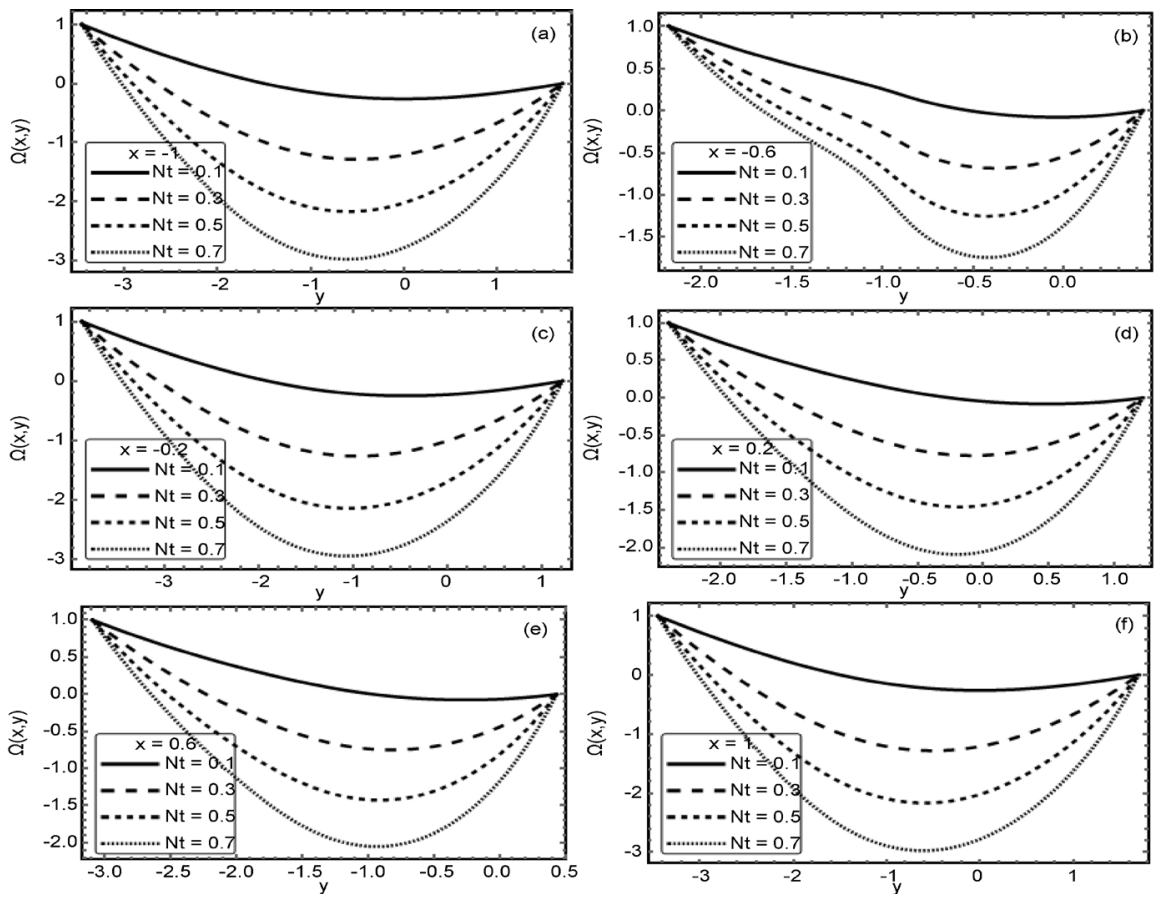

Figure 13. The concentration profile $\Omega$ is plotted against y for several values of $N t$ at $a=0.7, b=0.9, d=3, \phi=\frac{\pi}{3}, \operatorname{Pr}=3, E c=0.2, \quad W e=0.5, \quad m=1, \quad M=1.2$, $N b=0.7, n=3, G r=1, B r=2, s=2, F=1$, when (a) $x=-1$, (b) $x=-0.6$, (c) $x=$ -0.2 , (d) $x=0.2$, (e) $x=0.6$, (f) $x=1$. 

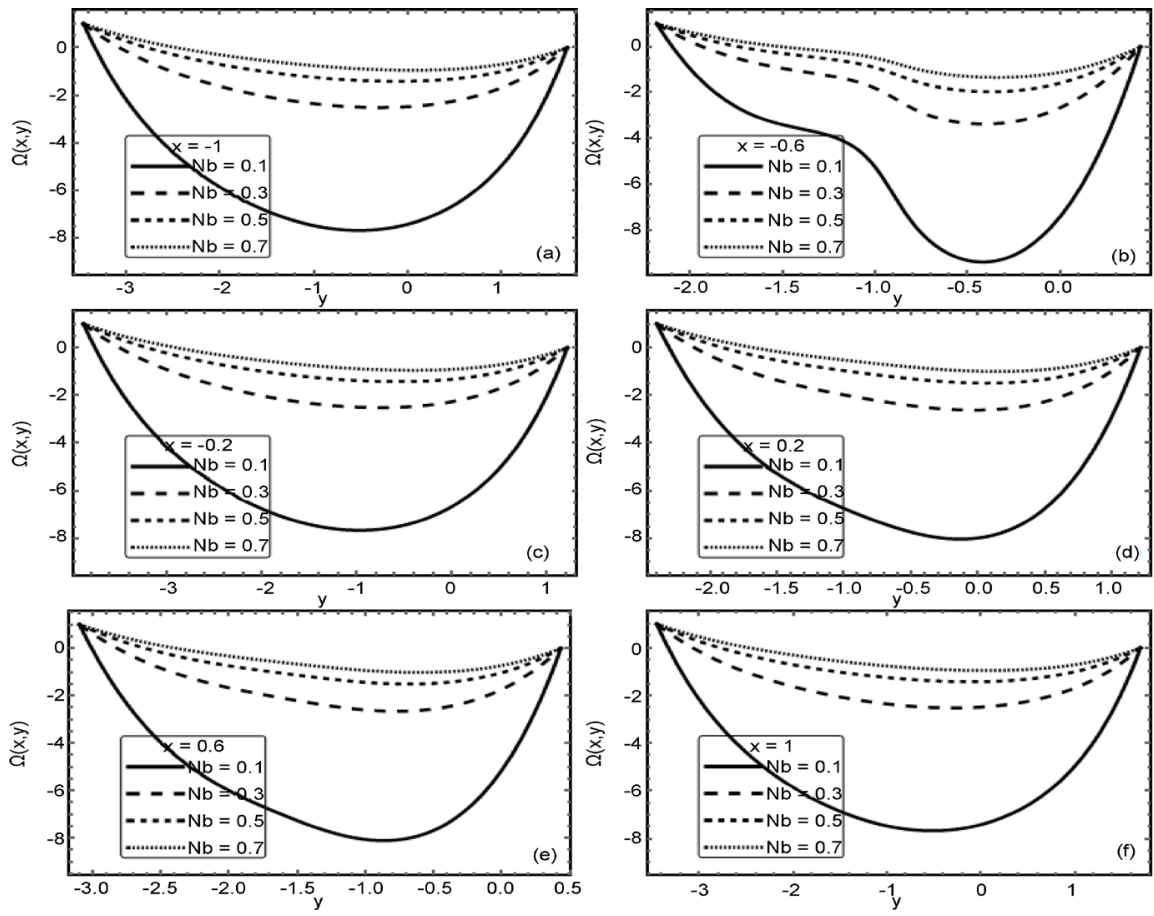

Figure 14. The concentration profile $\Omega$ is plotted against y for several values of $N b$ at $a=0.7, b=0.9, d=3, \phi=\frac{\pi}{3}, \operatorname{Pr}=3, E c=0.2, \quad W e=0.5, \quad m=1, \quad M=0.5$, $N_{t}=0.7, n=3, G r=1, B r=2, s=2, F=1$, when (a) $x=-1$, (b) $x=-0.6$, (c) $x=$ -0.2 , (d) $x=0.2$, (e) $x=0.6$, (f) $x=1$.
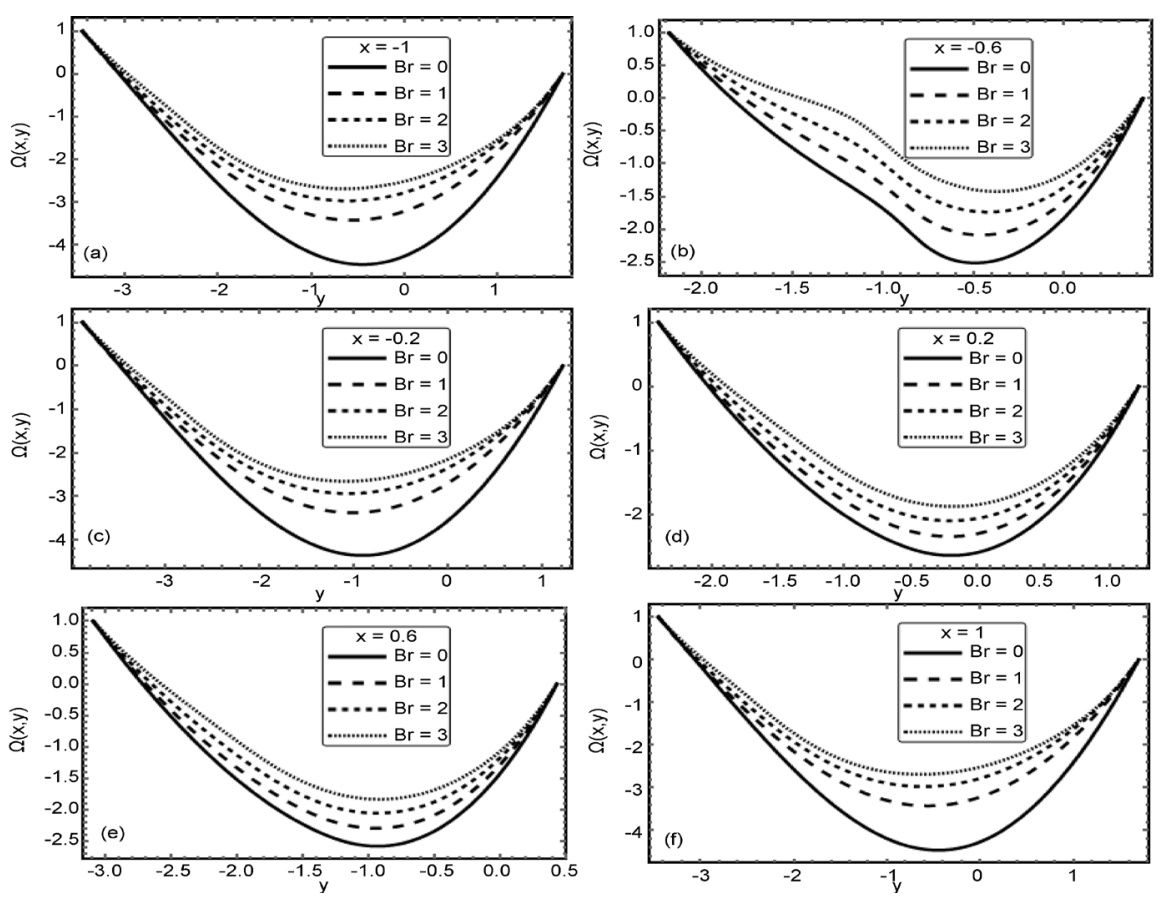

Figure 15. The concentration profile $\Omega$ is plotted against y for several values of $B r$ at $a=0.7, b=0.9, d=3, \phi=\frac{\pi}{3}, \operatorname{Pr}=3, \quad E c=0.2, \quad W e=0.5, \quad m=1, N_{t}=0.7$, $M=1.2, N b=0.7, n=3, G r=1, s=2, F=1$, when (a) $x=-1$, (b) $x=-0.6$, (c) $x=$ -0.2 , (d) $x=0.2$, (e) $x=0.6$, (f) $x=1$. 


\subsection{Pressure Gradient Profile}

Figures 16-21 examine the influence of the magnetic parameter $M$, the power law index $n$, the Weissenberg number we, the local nanoparticle Grashof

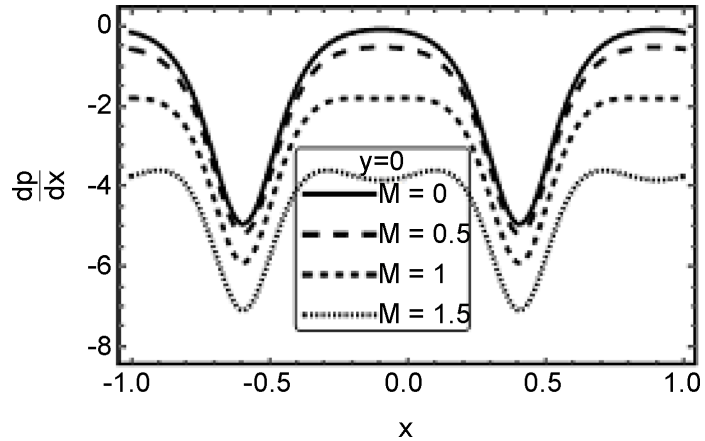

Figure 16. The pressure gradient $\frac{\mathrm{d} p}{\mathrm{~d} x}$ isplotted against $x$ for several values of $M$ at $a=0.7, \quad b=0.9, d=3, \phi=\frac{\pi}{3}, \operatorname{Pr}=3, \quad E c=0.2, \quad W e=0.5, \quad m=1, N_{t}=0.7$, $N b=0.7, n=3, G r=1, B r=2, s=2, F=1, y=0$.

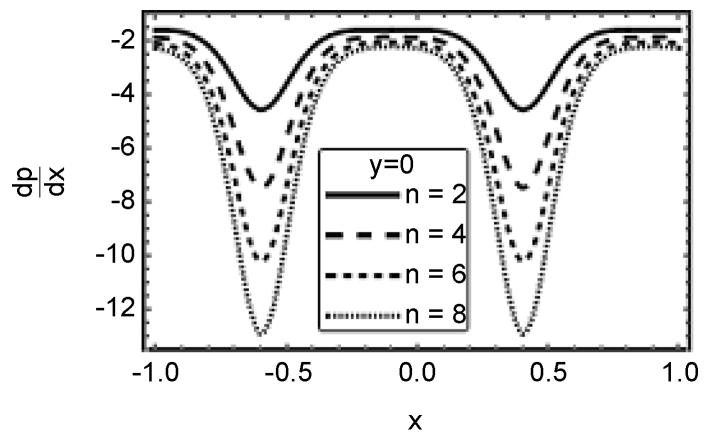

Figure 17. The pressure gradient $\frac{\mathrm{d} p}{\mathrm{~d} x}$ isplotted against $x$ for several values of $n$ at $a=0.7, b=0.9, d=3, \phi=\frac{\pi}{3}, \operatorname{Pr}=3, \quad E c=0.2, \quad W e=0.5, \quad m=1, N_{t}=0.7$, $N b=0.7, n=3, G r=1, B r=2, s=2, F=1, y=0$.

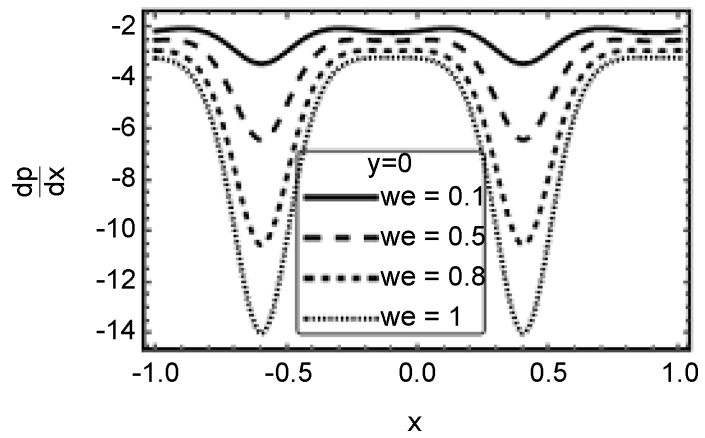

Figure 18. The pressure gradient $\frac{\mathrm{d} p}{\mathrm{~d} x}$ isplotted against $x$ for several values of $W e$ at $a=0.7, \quad b=0.9, \quad d=3, \quad \phi=\frac{\pi}{3}, \quad \operatorname{Pr}=3, \quad E c=0.2, \quad m=1, \quad M=1.2, \quad N_{t}=0.7$, $N b=0.7, n=3, G r=1, B r=2, s=2, F=1, y=0$. 


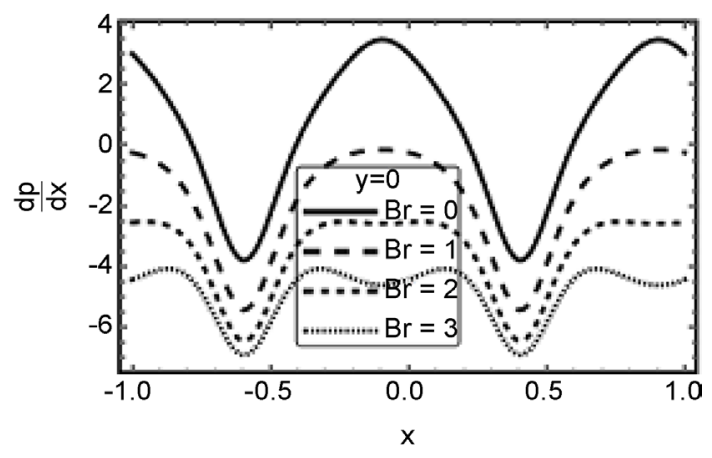

Figure 19. The pressure gradient $\frac{\mathrm{d} p}{\mathrm{~d} x}$ isplotted against $x$ for several values of $B r$ at $a=0.7, \quad b=0.9, \quad d=3, \quad \phi=\frac{\pi}{3}, \quad \operatorname{Pr}=3, \quad E c=0.2, \quad W e=0.5, \quad m=1, \quad M=1.2$, $N_{t}=0.7, N b=0.7, n=3, G r=1, s=2, \quad F=1, y=0$.

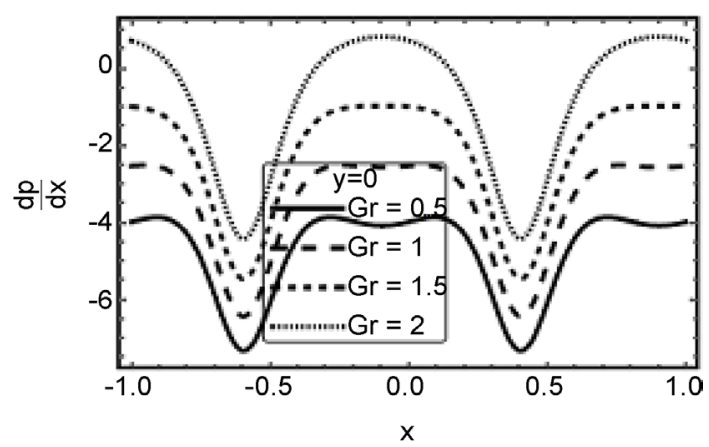

Figure 20. The pressure gradient $\frac{\mathrm{d} p}{\mathrm{~d} x}$ isplotted against $x$ for several values of $G r$ at $a=0.7, \quad b=0.9, \quad d=3, \quad \phi=\frac{\pi}{3}, \quad \operatorname{Pr}=3, \quad E c=0.2, \quad W e=0.5, \quad m=1, \quad M=1.2$, $N_{t}=0.7, \quad N b=0.7, n=3, B r=2, s=2, \quad F=1, y=0$.

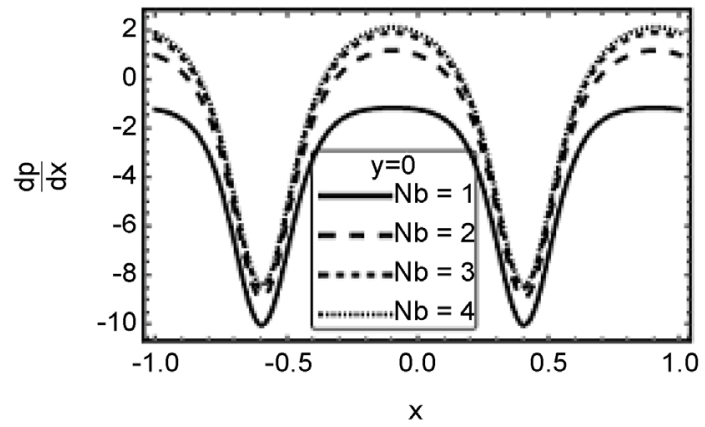

Figure 21. The pressure gradient $\frac{\mathrm{d} p}{\mathrm{~d} x}$ isplotted against $x$ for several values of $N b$ at $a=0.7, \quad b=0.9, \quad d=3, \quad \phi=\frac{\pi}{3}, \quad \operatorname{Pr}=3, \quad E c=0.2, \quad W e=0.5, \quad m=1, \quad M=1.2$, $N_{t}=0.7, n=3, G r=1, B r=2, s=2, F=1, y=0$.

number $b r$, the local temperature Grashof number $g_{r}$ and the Brownian motion parameter $N b$ on the pressure gradient. It has been observed that because of successive contraction and relaxation of peristaltic walls the pressure gradient 
shows oscillatory behavior. It is clear from Figures 16-19, that the magnitude of the pressure gradient decreases in view of an increase in $M, n$, we and $b r$. The situation is reversed in Figure 20 and Figure 21, the magnitude of the pressure gradient increases with an increase in $g r$ and $N b$. It is also noticed that the pressure gradient has its minimum values at the narrow parts of the channel and achieve its maximum values at the wider parts.

\section{Conclusions}

The peristaltic flow of a Carreau nanofluid through a porous medium with heat and mass transfer in the presence of Hall current, Joule heating and viscous dissipation is studied under assumption of long wavelength and low Reynolds number. The main results are summarized as follow:

- The velocity of Carraeu nanofluid increases at the neighborhood of the channel walls and decreases near the center of the channel by increasing the Weissenber number $W e$ and the power law index $n$.

- It is observed that the magnetic parameter $M$ and the thermophoresis parameter $n_{t}$ have opposite effects on the velocity to that of we and $n$.

- The temperature profile increases with an increase in the Brownian motion parameter $N b$ and decreases with an increase in the thermophoresis parameter $n_{t}$.

- The concetration profile decreases with an increase in the Brownian motion parameter $N b$ and increases with an increase in the thermophoresis parameter $n_{t}$.

- The pressure gradient decreases with the increase of the magnetic parameter $M$, the power law index $n$, the Weissenber number we and the local nanoparticle Grashof number $b r$, while it increases with the increase of the local temperature Grashof number $g_{r}$ and the thermophoresis parameter $n_{t}$.

\section{References}

[1] Choi, S.U.S. (1995) Enhancing Thermal Conductivity of Fluid with Nanoparticles. ASME Fluids Engineering Division, 231, 99-105.

[2] Choi, S.U.S., Zhang, Z.G., Yu, W., Lockwood, F.E. and Grulke, E.A. (2001) Anomalous Thermal Conductivity Enhancement in Nanotube Suspension. Applied Physics Letters, 79, 2252-2254. https://doi.org/10.1063/1.1408272

[3] Buongiorno, J. (2006) Convective Transport in Nanofluids. ASME Journal of Heat Transfer, 128, 240-250. https://doi.org/10.1115/1.2150834

[4] Anoop, K.B.T. (2009) Effects of Particle Size on the Convective Heat Transfer in Nanofluid in the Developing Region. International Journal of Heat and Mass transfer, 52, 2189-2195.

[5] Wang, X.Q. and Mujumdar, A.S. (2008) A Review on Nanofluids-Part II: Experiments and Applications. Brazilian Journal of Chemical Engineering, 25, 631-648. https://doi.org/10.1590/S0104-66322008000400002

[6] Kakaç, S. and Pramuanjaroenkij, A. (2009) Review of Convective Heat Transfer Enhancement with Nanofluids. International Journal of Heat and Mass Transfer, 52, 3187-3196. 
[7] Tzou, D.Y. (2008) Thermal Instability of Nanofluids. ASME Journal of Heat Transfer, 51, 2967-2979. https://doi.org/10.1016/j.ijheatmasstransfer.2007.09.014

[8] Yang, Y.Z., Zhang, G., Grulke, E.A., Anderson, W.B. and Wu, G. (2005) Heat Transfer Properties of Nanoparticle-in-Fluid Dispersions (Nanofluids) in Laminar Flow. International Journal of Heat and Mass Transfer, 48, 1107-1116.

[9] Latham, T.W. (1996) Fluid Motion in a Peristaltic Pump. Master's Thesis, Massachusetts Institute of Technology, Cambridge,

[10] Jaffrin, M.Y. and Shapiro, A.H. (1971) Peristaltic Pumping. Annual Review of Fluids Mechanics, 3, 13-36. https://doi.org/10.1146/annurev.fl.03.010171.000305

[11] Tripathi, A. and Chhabra, R.P. (1996) Transverse Laminar Flow of Non-Newtonian Fluid over a Bank of Long Cylinders. Chemical Engineering Communications, 147, 197-212. https://doi.org/10.1080/00986449608936504

[12] Sato, H., Kawai, T., Fujita, T. and Okabe, M. (2000) Two Dimensional Peristaltic Flow in Curved Channel. Transaction Japan Society of Mechanical Engineering, 66, 679-685. https://doi.org/10.1299/kikaib.66.679

[13] Mekheimer, K.S. (2003) Nonlinear Peristaltic Transport through a Porous meDium in an Inclined Planar Channel. Journal of Porous Media, 6, 189-201. https://doi.org/10.1615/JPorMedia.v6.i3.40

[14] Hameed, M. and Nadeem, S. (2007) Unsteady MHD Flow of a Non-Newtonian Fluid on a Porous Plate. Journal of Mathematical Analysis and Applications, 325, 724-733.

[15] Mekheimer, K.S. and Elmaboud, Y.A. (2008) The Influence of Heat Transfer of a Newtonain Fluid in a Vertical Annuls. Physical Letters A, 372, 1657-1665.

[16] Nadeem, S. and Akbar, N.S. (2009) Effects of Heat Transfer on the Peristaltic Transport of MHD Newtonian Fluid with Variable Viscosity: Application of Adomian Decomposition Method. Communications Nonlinear Science and Numerical Simulation, 14, 3844-3855.

[17] Srinivas, S. and Gayathri, R. (2009) Peristaltic Transport of a Newtonian Fluid in a Vertical Asymmetric Channel with Heat Transfer and Porous Medium. Applied Mathematics and Computation, 151, 185-196.

[18] El-Dabe, N.T., Ismail, G. and Darwesh, F.O. (2013) Peristaltic Transport of a Magneto Non-Newtonian Fluid through A Porous Medium in a Horizontal Finite Channel. IOSR Journal of Mathematics, 8, 32-39.

https://doi.org/10.9790/5728-0863239

[19] Akram, S. (2014) Effects of Nanofluids on Peristaltic Flow of a Carreau Fluid Model in an Inclined Magnetic Field. Heat Transfe-Asian Research, 43, 368-383. https://doi.org/10.1002/htj.21082

[20] Riaz, A., Ellahi, R. and Nadeem, S. (2014) Peristaltic Transport of a Carreau Fluid in a Compliant Rectangular Duct. Alexandria Engineering Journal, 53, 475-484.

[21] Nowar, K. (2014) Peristaltic Flow of a Nanofluid under the Effect of Hall Current and Porous Medium. Mathematical Problems in Engineering, 2014, 1-15. https://doi.org/10.1155/2014/389581

[22] Akber, N. S., Nadeem, S. and Khan, Z.H. (2014) Numerical Simulation of Peristaltic Flow of a Carreau Nanofluid in an Asymmetric Channel. Alexandria Engineering Journal, 53, 191-197.

[23] Hayat, T., Shafique, M., Tanveer A. and Alsaedi, A. (2016) Radiation Peristaltic Flow of Jeffery Nanofluid with Slip Conditions and Joule Heating. Plose One, 11, $1-11$. 
[24] Nadeem, S. and Akbar, N.S. (2010) Effects of Heat and Mass Transfer on Peristaltic Flow of Carreau Fliud in a Vertical Annulus, Zeitschrift fur Naturforschung-Section A. Journal of Physical Sciences, 65, 781-792.

[25] Nabil, T.M., El-Dabe, A.F. and Hussien, M.M. (2010) Magnetohydrodynamic Flow and Heat Transfer for a Peristaltic Motion of Carreau Fluid through a Porous Medium. Punjab University Journal of Mathematics, 42, 1-16.

[26] Nabil, T.M., El-Dabe, Agoor, B.M. and Alame, H. (2014) Peristaltic Motion of Non-Newtonian Fluid with Heat and Mass Transfer through a Porous Medium in Channel under Uniform Magnetic Field. Journal of Fluids, 2014, 1-12. https://doi.org/10.1155/2014/525769

[27] Nadeem, S. and Riaz, A. (2014) Effects of Heat and Mass Transfer on Peristaltic Flow of Nanofluid between Eccentric Cylinders. Applied Nanoscience, 4, 393-404. https://doi.org/10.1007/s13204-013-0225-X

[28] Tawfiq, N., El-Dabe, M., Ghaly, A.Y., Sallam, S.N., Elagamy, K. and Younis, Y.M. (2015) Peristaltic Pumping of a Conducting Sisko Fluid through Porous Medium with Heat and Mass Transfer. American Journal of Computational Mathematics, 5 , 304-315. https://doi.org/10.4236/ajcm.2015.53028

[29] Ramesh, K. and Devakar, M. (2015) Effects of Heat and Mass Transfer on the Peristaltic Transport of MHD Couple Stress Fluid through Porous Medium in a Vertical Asymmetric Channel. Journal of Fluids, 105, 1-19. https://doi.org/10.1155/2015/163832

[30] Bharali, A. and Borkakati, A.K. (1982) The Effect of Hall Currents on MHD Flow and Heat Transfer between Two Parallel Porous Plates. Applied Scientific Research, 39, 155-165. https://doi.org/10.1007/BF00457017

Submit or recommend next manuscript to SCIRP and we will provide best service for you:

Accepting pre-submission inquiries through Email, Facebook, LinkedIn, Twitter, etc. A wide selection of journals (inclusive of 9 subjects, more than 200 journals)

Providing 24-hour high-quality service

User-friendly online submission system

Fair and swift peer-review system

Efficient typesetting and proofreading procedure

Display of the result of downloads and visits, as well as the number of cited articles

Maximum dissemination of your research work

Submit your manuscript at: http://papersubmission.scirp.org/

Or contact ajcm@scirp.org 